\title{
Supertraces on the Algebras of Observables of the Rational Calogero Model with Harmonic Potential
}

\author{
S.E. Konstein and M.A. Vasiliev \\ I.E.Tamm Department of Theoretical Physics, P. N. Lebedev Physical Institute, \\ 117924 Leninsky Prospect 53, Moscow, Russia.
}

\begin{abstract}
We define a complete set of supertraces on the algebra $S H_{N}(\nu)$, the algebra of observables of the $N$-body rational Calogero model with harmonic interaction. This result extends the previously known results for the simplest cases of $N=1$ and $N=2$ to arbitrary $N$. It is shown that $S H_{N}(\nu)$ admits $q(N)$ independent supertraces where $q(N)$ is a number of partitions of $N$ into a sum of odd positive integers, so that $q(N)>1$ for $N \geq 3$. Some consequences of the existence of several independent supertraces of $S H_{N}(\nu)$ are discussed such as the existence of ideals in associated $W_{\infty}$ - type Lie superalgebras.
\end{abstract}

\section{Introduction}

In this paper we investigate some properties of the associative algebras which were shown in [1, 2, 3] to underly the rational Calogero model [4] and were denoted as $S H_{N}(\nu)$ in [5]. Algebra $S H_{N}(\nu)$ is the associative algebra of polynomials constructed from arbitrary elements $\sigma$ of the symmetric group $S_{N}$ and the generating elements $a_{i}^{\alpha}$ obeying the following relations

$$
\begin{gathered}
\sigma a_{i}^{\alpha}=a_{\sigma(i)}^{\alpha} \sigma, \\
{\left[a_{i}^{\alpha}, a_{j}^{\beta}\right]=\epsilon^{\alpha \beta} A_{i j},}
\end{gathered}
$$

where $i, j=1, \ldots, N, \alpha, \beta=0,1, \epsilon^{\alpha \beta}=-\epsilon^{\beta \alpha}, \epsilon^{01}=1$ and

$$
A_{i j}=\delta_{i j}+\nu \tilde{A}_{i j}, \quad \tilde{A}_{i j}=\delta_{i j} \sum_{l=1}^{N} K_{i l}-K_{i j} .
$$


Here $K_{i j} \in S_{N}$ with $i, j=1, \ldots, N, i \neq j$, are the elementary permutations $i \leftrightarrow j$ satisfying the relations

$$
K_{i j}=K_{j i}, K_{i j} K_{i j}=1, K_{i j} K_{j l}=K_{j l} K_{l i}=K_{l i} K_{i j}
$$

for $i \neq j \neq l \neq i$ and

$$
K_{i j} K_{k l}=K_{k l} K_{i j}
$$

if $i, j, k, l$ are pairwise different. Note that in this paper repeated Latin indices $i, j, k, \ldots$ do not imply summation.

The defining relations (11)-(3) are consistent. In particular, the Jacobi identities

$$
\left[a_{i}^{\alpha},\left[a_{j}^{\beta}, a_{k}^{\gamma}\right]\right]+\left[a_{j}^{\beta}\left[a_{k}^{\gamma}, a_{i}^{\alpha}\right]\right]+\left[a_{k}^{\gamma},\left[a_{i}^{\alpha}, a_{j}^{\beta}\right]\right]=0
$$

are satisfied.

An important property of $S H_{N}(\nu)$ which allows one to solve the Calogero model [4] is that this algebra possesses inner $s l_{2}$ automorphisms with the generators

$$
\begin{gathered}
T^{\alpha \beta}=\frac{1}{2} \sum_{i=1}^{N}\left(a_{i}^{\alpha} a_{i}^{\beta}+a_{i}^{\beta} a_{i}^{\alpha}\right), \\
{\left[T^{\alpha \beta}, T^{\gamma \delta}\right]=\epsilon^{\alpha \gamma} T^{\beta \delta}+\epsilon^{\alpha \delta} T^{\beta \gamma}+\epsilon^{\beta \gamma} T^{\alpha \delta}+\epsilon^{\beta \delta} T^{\alpha \gamma},}
\end{gathered}
$$

which act on the generating elements $a_{i}^{\alpha}$ as on $s l_{2}$ vectors

$$
\left[T^{\alpha \beta}, a_{i}^{\gamma}\right]=\epsilon^{\alpha \gamma} a_{i}^{\beta}+\epsilon^{\beta \gamma} a_{i}^{\alpha} .
$$

Calogero Hamiltonian is identified with the Cartan element $T^{01}$ which according to (7) induces $Z$ - gradation of $S H_{N}(\nu)$. The latter property allows one [2] to construct wave functions via the standard Fock procedure with the Fock vacuum $|0\rangle$ such that $a_{i}^{0}|0\rangle=0$. Thus, the elements $a_{i}^{\alpha}$ serve as generalized oscillators underlying the Calogero problem. The concrete realization of these oscillators in terms of Dunkl differential-difference operators [6] was presented in [1, 2].

These properties characterize the algebra $S H_{N}(\nu)$ as a natural generalization of the Heisenberg-Weyl algebra, the associative algebra of harmonic oscillators. Since the Lie algebra of quantum operators in the harmonic oscillator problem can be identified with the $W_{1+\infty}$ algebra [0], the Lie (super)algebras constructed from $S H_{N}(\nu)$ with the aid of supercommutators give rise to a class of the $W_{1+\infty}$ - type algebras which have been denoted as $W_{N, \infty}(\nu)$ in [8] where it was shown that all these algebras contain the Virasoro algebra as a subalgebra. The latter observation indicates that the algebras $S H_{N}(\nu)$ and $W_{N, \infty}(\nu)$ can be related to conformal models as well as to other classes of models in the range from quantum Hall effect [9] till higher-spin gauge theories [10] and KP hierarchy [11] where $W_{\infty}$ - type algebras prove to be important. An additional argument in favor of the relationship of $S H_{N}(\nu)$ to the quantum Hall effect is due to the known fact that the Calogero model can be interpreted as a one-dimensional reduction of the full anyonic problem [12, 3].

For lower values of $N$, a nature of $S H_{N}(\nu)$ is rather well understood. $S H_{1}(\nu)$ is the ordinary Heisenberg-Weyl algebra (since $\nu$ - dependence is artificial in this case we will use 
the notation $S H_{1}$ ). Properties of this algebra are very well studied (see e.g. [13]). Note that since the center of mass coordinates $1 / N \sum_{i=1}^{N} a_{i}^{\alpha}$ decouple from everything else in the defining relations (11)-(3), the associative algebra $S H_{N}(\nu)$ has the structure $S H_{N}(\nu)=$ $S H_{1} \otimes S H_{N}^{\prime}(\nu)$ where, by definition, $S H_{N}^{\prime}(\nu)$ is the algebra of elements depending only on the relative coordinates $a_{i}^{\alpha}-a_{j}^{\alpha}$.

The properties of $S H_{2}^{\prime}(\nu)$ are well studied too [14. The algebra $S H_{2}^{\prime}(\nu)$ is defined by the relations

$$
\left[a^{\alpha}, a^{\beta}\right]=\epsilon^{\alpha \beta}(1+2 \nu K),
$$

where $K$ is the only nontrivial element of $S_{2}$ while $a^{\alpha}$ are the relative motion oscillators. For the particular case of $\nu=0$ one recovers the algebra $S H_{1}$ in the sector of the $K$ independent elements.

In [14 it was shown that $S H_{2}^{\prime}(\nu)$ admits a unique supertrace operation defined by the simple formula

$$
\operatorname{str}(1)=1, \quad \operatorname{str}(K)=-2 \nu, \quad \operatorname{str}(W)=\operatorname{str}(W K)=0
$$

for any polynomial $W \in S H_{2}^{\prime}$ of the form

$$
W=\sum_{n=1}^{\infty} W_{\alpha_{1} \ldots \alpha_{n}} a^{\alpha_{1}} \ldots a^{\alpha_{n}}
$$

with arbitrary totally symmetric multispinors $W_{\alpha_{1} \ldots \alpha_{n}}$. For the particular case of $\nu=0$ one recovers the supertrace on $S H_{1}$.

Furthermore it was shown in [14] by explicit evaluation of the invariant bilinear form $B(x, y) \stackrel{\text { def }}{=} \operatorname{str}(x y)$ that for $\nu=l+\frac{1}{2}\left(l\right.$ is any integer) $S H_{2}^{\prime}(\nu)$ reduces to finite-dimensional matrix algebras up to some infinite-dimensional ideals $\mathcal{I}$ which decouple from everything under the supertrace operation (9), i.e. $\operatorname{str}(x y)=0, \forall x \in \mathcal{I}$.

In [15] it was then observed that $S H_{2}^{\prime}(\nu)$ is isomorphic to the factor of the enveloping algebra $U(\operatorname{osp}(1 ; 2))$ of $\operatorname{ssp}(1 ; 2)$ over its ideal generated by the quadratic Casimir operator $C_{2}$ by factoring out all elements of the form $\left(C_{2}-c_{2}\right) U(\operatorname{osp}(1 ; 2))$ where $c_{2}=\frac{1}{16}\left(4 \nu^{2}-1\right)$ is an arbitrary constant. In its turn this observation clarified the origin of the ideals of $S H_{2}^{\prime}(\nu)$ at $\nu=l+\frac{1}{2}$ as corresponding to the finite-dimensional representations of $\operatorname{osp}(1 ; 2)$.

Although the algebra $S H_{N}(\nu)$ is getting interesting applications for any $N$, till now understanding of its algebraic properties for $N>2$ is far from being satisfactory. In particular there is no interpretation of $S H_{N}(\nu)$ in terms of enveloping algebras of finitedimensional superalgebras and nothing is known about ideals of $S H_{N}(\nu)$ which information is very important in applications.

In this paper we analyze the existence of the invariant supertrace operation on $S H_{N}(\nu)$ i.e. such a complex valued linear function $\operatorname{str}(f)$ on $S H_{N}(\nu)$ that

$$
\operatorname{str}([f, g\})=0, \quad \forall f, g \in S H_{N}(\nu)
$$

with the convention that

$$
[f, g\}=f g-(-1)^{\pi(f) \pi(g)} g f,
$$

where the parity $\pi$ in $S H_{N}(\nu)$ is defined as follows:

$$
\pi\left(a_{i}^{\alpha}\right)=1, \quad \pi\left(K_{i j}\right)=0 .
$$


Let us note that an attempt to define differently graded traces like, e.g., an ordinary trace $(\pi \equiv 0)$ unlikely leads to interesting results.

Knowledge of the supertrace operations on $S H_{N}(\nu)$ is useful in various respects. One of the most important applications of the supertrace is that it gives rise to $n$-linear invariant forms

$$
\operatorname{str}\left(a_{1} a_{2} \ldots a_{n}\right)
$$

that allows one to work with the algebra essentially in the same way as with the ordinary finite-dimensional matrix algebras and, for example, construct Lagrangians when working with dynamical theories based on $S H_{N}(\nu)$. Another useful property is that since null vectors of any invariant bilinear form span a both-side ideal of the algebra, this gives a powerful device for investigating ideals which decouple from everything under the supertrace operation as it happens in $S_{2}(\nu)$ for half-integer $\nu$. It is also worth mentioning that having an explicit form of the trilinear form in one or another basis is practically equivalent to defining a star-product law in the algebra.

An important motivation for the analysis of the supertraces of $S H_{N}(\nu)$ is due to its deep relationship with the analysis of the representations of this algebra, which in its turn gets applications to the analysis of the wave functions of the Calogero model. For example, given representation of $S H_{N}(\nu)$, one can speculate that it induces some supertrace on this algebra as (appropriately regularized) supertrace of (infinite) representation matrices. When the corresponding bilinear form degenerates this would imply that the representation becomes reducible.

As we show, the situation for $S H_{N}(\nu)$ is very interesting since starting from $N=3$ it admits more than one independent supertrace in contrast to the cases of $N=1$ and $N=2$. This fact is in agreement with the results of [5] where it was shown that there exist many inequivalent lowest-weight type representations of $S H_{N}(\nu)$ for higher $N$ (these representations are classified according to the representations of $S_{N}$.) Another important consequence of this phenomenon is that the Lie superalgebras $W_{N, \infty}(\nu)$ are not simple while appropriate their simple subalgebras possess non-trivial outer automorphisms.

The paper is organized as follows. In Section \we analyze consequences of $S_{N}$ and $s l_{2}$ automorphisms of $S H_{N}(\nu)$. In Section [II] we discuss general properties of the supertraces and consequences of the existence of several independent supertraces. In Section $\mathbb{I V}$ we study the restrictions on supertraces of the group algebra of $S_{N}$ considered as a subalgebra of $S H_{N}(\nu)$, which follow from the defining relations of $S H_{N}(\nu)$. These restrictions are called ground level conditions $(G L C)$. They play a fundamental role in the problem since as we show in Section $\mathrm{V}$ every solution of $G L C$ admits a unique extension to some supertrace on $S H_{N}(\nu)$. In Appendix $\mathrm{A}$ it is shown that the number of independent supertraces on $S H_{N}(\nu)$ equals to the number of partitions of $N$ into a sum of odd positive integers. Some technical details of the proof of Section $\mathrm{V}$ are collected in Appendices $\mathrm{B}$ and $\mathrm{C}$.

\section{Finite-Dimensional Groups of Automorphisms}

The group algebra of $S_{N}$ is the finite-dimensional subalgebra of $S H_{N}(\nu)$. The elements $\sigma \in S_{N}$ induce inner automorphisms of $S H_{N}(\nu)$. It is well known, that any $\sigma \in S_{N}$ can 
be expanded into a product of pairwise commuting cycles

$$
\sigma=c_{1} c_{2} c_{3} \ldots c_{t}
$$

where $c_{\mathfrak{w}}, \mathfrak{w}=1, \ldots, t$, are cyclic permutations acting on distinct subsets of values of indices $i$. For example, a cycle which acts on the first $s$ indices as $1 \rightarrow 2 \rightarrow \ldots \rightarrow s \rightarrow 1$ has the form

$$
c=K_{12} K_{23} \ldots K_{(s-1) s} \cdot
$$

We use the notation $|c|$ for the length of the cycle $c$. For the cycle (16), $|c|=s$. We take a convention that the cycles of unit length are associated with all values of $i$ such that $\sigma(i)=i$, so that the relation $\sum_{\mathfrak{w}}\left|c_{\mathfrak{w}}\right|=N$ is true.

Given permutation $\sigma \in S_{N}$, we introduce a new set of basis elements $\mathfrak{B}_{\sigma}=\left\{b^{I}\right\}$ instead of $\left\{a_{i}^{\alpha}\right\}$ in the following way. For every cycle $c_{\mathfrak{w}}$ in the decomposition (15) $(\mathfrak{w}=1, \ldots, t)$, let us fix some index $l_{\mathfrak{w}}$, which belongs to the subset associated with the cycle $c_{\mathfrak{w}}$. The basis elements $b_{\mathfrak{w} j}^{\alpha}, j=1, \ldots,\left|c_{\mathfrak{w}}\right|$, which realize 1 -dimensional representations of the commutative cyclic group generated by $c_{\mathfrak{w}}$, have the form

$$
b_{\mathfrak{w} j}^{\alpha}=\frac{1}{\sqrt{\left|c_{\mathfrak{w}}\right|}} \sum_{k=1}^{\left|c_{\mathfrak{w}}\right|}\left(\lambda_{\mathfrak{w}}\right)^{j k} a_{l(\mathfrak{w}, k)}^{\alpha},
$$

where $l(\mathfrak{w}, k)=c_{\mathfrak{w}}^{-k}\left(l_{\mathfrak{w}}\right)$ and

$$
\lambda_{\mathfrak{w}}=\exp \left(2 \pi i /\left|c_{\mathfrak{w}}\right|\right) .
$$

From the definition (17) it follows that

$$
\begin{gathered}
c_{\mathfrak{w}} b_{\mathfrak{w} j}^{\alpha}=\left(\lambda_{\mathfrak{w}}\right)^{j} b_{\mathfrak{w} j}^{\alpha} c_{\mathfrak{w}}, \\
c_{\mathfrak{w}} b_{\mathfrak{n} j}^{\alpha}=b_{\mathfrak{n} j}^{\alpha} c_{\mathfrak{w}}, \text { for } \mathfrak{n} \neq \mathfrak{w}
\end{gathered}
$$

and therefore

$$
\sigma b_{\mathfrak{w} j}^{\alpha}=\left(\lambda_{\mathfrak{w}}\right)^{j} b_{\mathfrak{w} j}^{\alpha} \sigma .
$$

In what follows, instead of writing $b_{\mathfrak{w} j}^{\alpha}$ we use the notation $b^{I}$ with the label $I$ accounting for the full information about the index $\alpha$, the index $\mathfrak{w}$ enumerating cycles in (15), and the index $j$ which enumerates various elements $b_{\mathfrak{w} j}^{\alpha}$ related to the cycle $c_{\mathfrak{w}}$, i.e. $I(I=1, \ldots, 2 N)$ enumerates all possible triples $\{\alpha, \mathfrak{w}, j\}$. We denote the index $\alpha$, the cycle and the eigenvalue in (19) corresponding to some fixed index $I$ as $\alpha(I), c(I)$, and $\lambda_{I}=\left(\lambda_{\mathfrak{w}}\right)^{j}$, respectively. The notation $\sigma(I)=\sigma_{0}$ implies that $b^{I} \in \mathfrak{B}_{\sigma_{0}} \cdot \mathfrak{B}_{1}$ is the original basis of the generating elements $a_{i}^{\alpha}$ (here $\mathbf{1}$ is the unit permutation).

Let $\mathfrak{M}(\sigma)$ be the matrix which maps $\mathfrak{B}_{1} \longrightarrow \mathfrak{B}_{\sigma}$ in accordance with (17),

$$
b^{I}=\sum_{i, \alpha} \mathfrak{M}_{i \alpha}^{I}(\sigma) a_{i}^{\alpha} .
$$

Obviously this mapping is invertible. Using the matrix notations one can rewrite (21) as

$$
\sigma b^{I} \sigma^{-1}=\sum_{J=1}^{2 N} \Lambda_{J}^{I}(\sigma) b^{J}, \quad \forall b^{I} \in \mathfrak{B}_{\sigma},
$$


where $\Lambda_{I}^{J}(\sigma)=\delta_{I}^{J} \lambda_{I}$.

Every polynomial in $S H_{N}(\nu)$ can be expanded into a sum of monomials of the form

$$
b^{I_{1}} b^{I_{2}} \ldots b^{I_{s}} \sigma,
$$

where all $\sigma\left(I_{k}\right)=\sigma$. Every monomial of this form realizes some one-dimensional representation of the Abelian group generated by all cycles $c_{\mathfrak{w}}$ in the decomposition (15).

The commutation relations for the generating elements $b^{I}$ follow from (2) and (3)

$$
\left[b^{I}, b^{J}\right]=F^{I J}=\mathcal{C}^{I J}+\nu f^{I J},
$$

where

$$
\mathcal{C}^{I J}=\epsilon^{\alpha(I) \alpha(J)} \delta_{c(I) c(J)} \delta_{\lambda_{I} \lambda_{J}^{-1}}
$$

and

$$
f^{I J}=\sum_{i, j, \alpha, \beta} \mathfrak{M}_{i \alpha}^{I}(\sigma) \mathfrak{M}_{j \beta}^{J}(\sigma) \epsilon^{\alpha \beta} \tilde{A}_{i j} .
$$

The indices $I, J$ are raised and lowered with the aid of the symplectic form $\mathcal{C}^{I J}$

$$
\mu^{I}=\sum_{J} \mathcal{C}^{I J} \mu_{J}, \quad \mu_{I}=\sum_{J} \mu^{J} \mathcal{C}_{J I} ; \quad \sum_{M} \mathcal{C}_{I M} \mathcal{C}^{M J}=-\delta_{I}^{J} .
$$

Note that the elements $b^{I}$ are normalized in (17) in such a way that the $\nu$-independent part in (25) has the form (26).

Another important finite-dimensional algebra of inner automorphisms of $S H_{N}(\nu)$ is the $s l_{2}$ algebra which acts on the indices $\alpha$. It is spanned by the $S_{N}$-invariant secondorder polynomials (5). Evidently, $S H_{N}(\nu)$ decomposes into the infinite direct sum of only finite-dimensional irreducible representations of this $s l_{2}$ spanned by various homogeneous polynomials (24).

From the defining relations (11)-(3) it follows that $S H_{N}(\nu)$ is $Z_{2}$ - graded with respect to the automorphism

$$
f\left(a_{j}^{\alpha}\right)=-a_{j}^{\alpha}, \quad f\left(K_{i j}\right)=K_{i j}
$$

which gives rise to the parity $\pi$ (13). In applications to higher-spin models, this automorphism distinguishes between bosons and fermions.

The algebra $S H_{N}(\nu)$ admits the antiautomorphism $\rho$,

$$
\rho\left(a_{k}^{\alpha}\right)=i a_{k}^{\alpha}, \quad \rho\left(K_{i j}\right)=K_{i j},
$$

which leaves invariant the basic relations (11)-(3) provided that an order of operators is reversed according to the defining property of antiautomorphisms: $\rho(A B)=\rho(B) \rho(A)$. From (15), (16) and (21) it follows that

$$
\rho(\sigma)=\sigma^{-1}, \quad \rho\left(b^{I}\right)=i b^{J},
$$

where $J$ is related to $I$ in such a way that $\alpha(J)=\alpha(I), \sigma(J)=(\sigma(I))^{-1}, c(J)=(c(I))^{-1}$ and $\lambda_{J}=\lambda_{I}^{-1}$. Note that in higher-spin theories the counterpart of $\rho$ distinguishes between odd and even spins [16]. 


\section{General Properties of Supertrace}

In this section we summarize some general properties to be respected by any supertrace in $S H_{N}(\nu)$.

Let $A$ be an arbitrary associative $Z_{2}$ graded algebra with the parity function $\pi(x)=0$ or 1. Suppose that $A$ admits some supertrace operations $s t r_{p}$ where the label $p$ enumerates different nontrivial supertraces. We call a supertrace $\operatorname{str}$ even (odd) if $\operatorname{str}(x)=0 \forall x \in A$ such that $\pi(x)=1(0)$. Let $T_{A}$ be a linear space of supertraces on $A$. We say that $\operatorname{dim} T_{A}$ is the number of supertraces on $A$.

Given parity-preserving (anti)automorphism $\tau$ and supertrace operation str on $A$, $\operatorname{str}(\tau(x))$ is some supertrace as well. For inner automorphisms $\tau\left(\tau(x)=p x p^{-1}, \pi(p)=0\right)$ it follows from the defining property of the supertrace that $\operatorname{str}(\tau(x))=\operatorname{str}(x)$. Thus, $T_{A}$ forms a representation of the factor-group of the parity preserving automorphisms and antiautomorphisms of $A$ over the normal subgroup of the inner automorphisms of $A$. Applying this fact to the original parity automorphism $(-1)^{\pi}$ one concludes that $T_{A}$ can always be decomposed into a direct sum of subspaces of even and odd supertraces, $T_{A}=T_{A}^{0} \oplus T_{A}^{1}$ and that $T_{A}^{1}=0$ if the parity automorphism is inner.

In the sequel we only consider the case where $\operatorname{dim} T_{A}<\infty$ and there are no nontrivial odd supertraces. Let $A=A_{1} \otimes A_{2}$ with the associative algebras $A_{1}$ and $A_{2}$ endowed with some even supertrace operations $t_{1}$ and $t_{2}$, respectively. The supertrace on $A$ can be defined by setting $\operatorname{str}\left(a_{1} \otimes a_{2}\right)=t_{1}\left(a_{1}\right) t_{2}\left(a_{2}\right), \forall a_{1} \in A_{1}, \forall a_{2} \in A_{2}$. As a result, one concludes that $T_{A}=T_{A_{1}} \otimes T_{A_{2}}$. In the case of $S H_{N}(\nu)$ one thus can always separate out a contribution of the center of mass coordinates as an overall factor $\left(\mathrm{SH}_{1}\right.$ admits the unique supertrace).

If $A$ is finite-dimensional then the existence of two different supertraces indicates that $A$ admits non-trivial both-side ideals. Actually, consider the bilinear form $B(f, g)=$ $\alpha_{1} \operatorname{str}_{1}(f g)+\alpha_{2} \operatorname{str}_{2}(f g)$ with arbitrary parameters $\alpha_{1}, \alpha_{2} \in \mathbb{C}$ and elements $f, g \in A$. The determinant of this bilinear form is some polynomial of $\alpha_{1}$ and $\alpha_{2}$. Therefore it vanishes for certain ratios $\alpha_{1} / \alpha_{2}$ or $\alpha_{2} / \alpha_{1}$ according to the central theorem of algebra. Thus, for these values of the parameters the bilinear form $B$ degenerates and admits non-trivial null vectors $x, B(x, g)=0, \forall g \in A$. It is easy to see that the linear space $\mathcal{I}$ of all null vectors $x$ is some both-side ideal of $A$. For infinite-dimensional algebras the existence of several supertraces does not necessarily imply the existence of ideals. As mentioned in introduction the existence of several supertrace operations may be related to the existence of inequivalent representations. Also it is worth mentioning that for the case of infinite-dimensional algebras and representations under investigation it can be difficult to use the standard (i.e. matrixwise) definition of the supertrace. In this situation the formal definition of the supertraces on the algebra we implement in this paper is the only rigorous one.

Let $l_{A}$ be the Lie superalgebra which is isomorphic to $A$ as a linear space and is endowed with the product law (12). It contains the subalgebra $s l_{A} \in l_{A}$ spanned by elements $g$ such that $\operatorname{str}_{p}(g)=0$ for all $p$. Evidently $s l_{A}$ forms the ideal of $l_{A}$. The factor algebra $t_{A}=l_{A} / s l_{A}$ is a commutative Lie algebra isomorphic to $T_{A}^{*}$ as a linear space. Elements of $t_{A}$ different from the unit element of $A$ (which exist if $\operatorname{dim} T_{A}>1$ ) can induce outer automorphisms of $s l_{A}$. Let us note that it is this $s l_{A}$ Lie superalgebra which usually has 
physical applications. For the case of $S H_{N}(\nu)$ under consideration the algebra $l_{S H_{N}(\nu)}$ is identified with the algebra $W_{N, \infty}(\nu)$ introduced in [8]. We therefore conclude that these algebras are not simple for $N>2$ because it is shown below that $S H_{N}(\nu)$ admits several supertraces for $N>2$. Instead one can consider the algebras $s W_{N, \infty}(\nu)$.

Let $l_{A}$ contain some subalgebra $\mathcal{L}$ such that $A$ decomposes into a direct sum of irreducible representations of $\mathcal{L}$ with respect to the adjoint action of $\mathcal{L}$ on $A$ via supercommutators. Then, only trivial representations of $\mathcal{L}$ can contribute to any supertrace on $A$. Actually, consider some non-trivial irreducible representation $R$ of $\mathcal{L}$. Any $r \in R$ can be represented as

$$
r=\sum_{j}\left[l_{j}, r_{j}\right\}, \quad l_{j} \in \mathcal{L}, \quad r_{j} \in R
$$

since elements of the form (32) span the invariant subspace in $R$. From (11) it follows then that $\operatorname{str}(r)=0, \forall r \in R$.

From the definition of the supertrace it follows that

$$
\operatorname{str}\left(a_{1} a_{2}\right)+\operatorname{str}\left(a_{2} a_{1}\right)=0
$$

for arbitrary odd elements $a_{1}$ and $a_{2}$ of $A$. A simple consequence of this relation is that

$$
\operatorname{str}\left(a_{1} a_{2} \ldots a_{n}+a_{2} \ldots a_{n} a_{1}+\ldots+a_{n} a_{1} \ldots a_{n-1}\right)=0
$$

is true for an arbitrary even $n$ if all $a_{i}$ are some odd elements of $A$. Since we assume that the supertrace is even (34) is true for any $n$. This simple property turns out to be practically useful because, when odd generating elements are subject to some commutation relations with the right hand sides expressed via even generating elements like in (2), it often allows one to reduce evaluation of the supertrace of a degree- $n$ polynomial of $a_{i}$ to supertraces of lower degree polynomials.

Another useful property is that in order to show that the characteristic property of the supertrace (11) is true for any $x, g \in A$, it suffices to show this for a particular case where $x$ is arbitrary while $g$ is an arbitrary generating element of some fixed system of generating elements. Then (11) for general $x$ and $g$ will follow from the properties that $A$ is associative and str is linear. For the particular case of $S H_{N}(\nu)$ this means that it is enough to set either $g=a_{i}^{\alpha}$ or $g=K_{i j}$. $A$.

Let us now turn to some specific properties of $S H_{N}(\nu)$ as a particular realization of

By identifying $\mathcal{L}$ with $s l_{2}(5)$ and taking into account that $S H_{N}(\nu)$ decomposes into a direct sum of irreducible finite-dimensional representations of $s l_{2}$, one arrives at the following

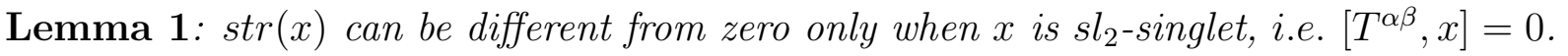
Corollary: Any supertrace on $S H_{N}(\nu)$ is even.

Analogously one deduces consequences of the $S_{N}$ symmetry. In particular, one proves Lemma 2: Given $c \in S_{N}$ such that $c F=\mu F c$ for some element $F$ and any constant $\mu \neq 1, \operatorname{str}(F)=0$. Given monomial $F=b^{I_{1}} b^{I_{2}} \ldots b^{I_{s}} \sigma$ with $b^{I_{k}} \in \mathfrak{B}_{\sigma}$ and a cycle $c_{0}$ in the decomposition (15) of $\sigma$ one concludes that $\operatorname{str}(F)=0$ if $\prod_{k: c\left(I_{k}\right)=c_{0}} \lambda_{I_{k}} \neq 1$ where $\lambda_{I_{k}}$ are the eigenvalues (21) of $b^{I_{k}}$. 


\section{Ground Level Conditions}

Let us analyze restrictions on a form of $\operatorname{str}(a), a \in S_{N}$, which follow from the defining relations of $S H_{N}(\nu)$.

Firstly, we describe supertraces on the group algebra of $S_{N}$. Let some permutation $\sigma$ decomposes into $n_{1}$ cycles of length $1, n_{2}$ cycles of length $2, \ldots$ and $n_{N}$ cycles of length $N$. The non-negative integers $n_{k}$ satisfy the relation

$$
\sum_{k=1}^{N} k n_{k}=N
$$

and fix $\sigma$ up to some conjugation $\sigma \rightarrow \tau \sigma \tau^{-1}, \tau \in S_{N}$. Thus

$$
\operatorname{str}(\sigma)=\varphi\left(n_{1}, n_{2}, \ldots, n_{N}\right),
$$

where $\varphi\left(n_{1}, n_{2}, \ldots, n_{N}\right)$ is an arbitrary function. Obviously the linear space of invariant functions on $S_{N}$ (i.e. such that $\left.f\left(\tau \sigma \tau^{-1}\right)=f(\sigma)\right)$ coincides with the linear space of supertraces on the group algebra of $S_{N}$. Therefore, the dimension of the linear space of supertraces is equal to the number $p(N)$ of independent solutions of (35), the number of conjugacy classes of $S_{N}$. One can introduce the generating function for $p(N)$ as $P(q)=$ $\sum_{n=0}^{\infty} p(n) q^{n}=\prod_{k=1}^{\infty} \frac{1}{\left(1-q^{k}\right)}$. The properties of this generating function and of the quantities $p(N)$ are discussed in details e.g. in [17].

According to the general argument of the previous section, the existence of several independent traces implies that the group algebra of $S_{N}$ must have some ideals. Indeed it can be shown to decompose into a direct sum of matrix algebras $\mathrm{Mat}_{n}$.

Since the group algebra of $S_{N}$ is embedded into $S H_{N}(\nu)$ some additional restrictions on the functions $\varphi\left(n_{1}, n_{2}, \ldots, n_{N}\right)$ follow from (11) and the defining relations (2)-(3) of $S H_{N}(\nu)$. Actually, consider some elements $b^{I}$ such that $\lambda_{I}=-1$. Then, one finds from (11) and (21) that $\operatorname{str}\left(b^{I} b^{J} \sigma\right)=-\operatorname{str}\left(b^{J} \sigma b^{I}\right)=\operatorname{str}\left(b^{J} b^{I} \sigma\right)$ and therefore

$$
\operatorname{str}\left(\left[b^{I}, b^{J}\right] \sigma\right)=0 .
$$

Since these conditions restrict supertraces of degree- 0 polynomials of $a_{i}^{\alpha}$ we call them ground level conditions $(G L C)$. Thus for every permutation $\sigma$ and any even integer $2 k$ such that there exists some cycle $c$ of length $|c|=2 k$ in the decomposition (15) we have $G L C$ (37) with $b^{I}$ such that $c(I)=c$. Note however that if $\lambda_{J} \neq-1$ or $c(J) \neq c(I)$ then the relation $\operatorname{str}\left(\left[b^{I}, b^{J}\right] \sigma\right)=0$ is trivially satisfied as a consequence of Lemma 2 .

It is convenient to rewrite $G L C$ in the following form

$$
\operatorname{str}\left(c_{0} \sigma_{0}\right)=-\operatorname{str}\left(\left(\left[b_{0 k}^{0}, b_{0 k}^{1}\right]-1\right) c_{0} \sigma_{0}\right),
$$

where $c_{0}$ is any cycle of even length $2 k$ in the decomposition of the permutation $\sigma=c_{0} \sigma_{0}$ and $b_{0 k}^{\alpha}$ is the corresponding variable (17) with $\left(\lambda_{0}\right)^{k}=-1$, i.e. $c_{0} b_{0 k}^{\alpha}=-b_{0 k}^{\alpha} c_{0}, \sigma_{0} b_{0 k}^{\alpha}=$ $b_{0 k}^{\alpha} \sigma_{0}$ and $\lambda_{0}=\exp \left(2 \pi i /\left|c_{0}\right|\right)$.

To work out the explicit form of the restrictions on the functions $\varphi\left(n_{1}, n_{2}, \ldots n_{N}\right)$ which follow from $G L C$ one has to use the following simple facts from the theory of the symmetric group: 
Lemma 3: Let $c_{1}$ and $c_{2}$ be two distinct cycles in the decomposition (15). Let indices $i_{1}$ and $i_{2}$ belong to the subsets of indices associated with the cycles $c_{1}$ and $c_{2}$, respectively. Then the permutation $c=c_{1} c_{2} K_{i_{1} i_{2}}$ is a cycle of length $|c|=\left|c_{1}\right|+\left|c_{2}\right|$.

Lemma 4: Given cyclic permutation $c \in S_{N}$, let $i \neq j$ be two indices such that $c^{k}(i)=j$, where $k$ is some positive integer, $k<|c|$. Then $c K_{i j}=c_{1} c_{2}$ where $c_{1,2}$ are some noncoinciding mutually commuting cycles such that $\left|c_{1}\right|=k$ and $\left|c_{2}\right|=|c|-k$.

Using the definition (17), the commutation relations (11)-(3) and Lemmas 3 and 4 one reduces $G L C$ to the following system of equations:

$$
\begin{aligned}
& n_{2 k} \varphi\left(n_{1}, \ldots, n_{2 k}, \ldots, n_{N}\right) \\
& =-\nu n_{2 k}\left(2 \sum_{s \neq k, s=1}^{2 k-1} O_{s} \varphi\left(n_{1}, \ldots, n_{s}+1, \ldots, n_{2 k-s}+1, \ldots, n_{2 k}-1, \ldots, n_{N}\right)\right. \\
& +2 O_{k} \varphi\left(n_{1}, \ldots, n_{k}+2, \ldots, n_{2 k}-1, \ldots, n_{N}\right) \\
& +\sum_{s \neq 2 k ; s=1}^{N} s n_{s} \varphi\left(n_{1}, \ldots, n_{s}-1, \ldots, n_{2 k}-1, \ldots, n_{2 k+s}+1, \ldots, n_{N}\right) \\
& \left.+2 k\left(n_{2 k}-1\right) \varphi\left(n_{1}, \ldots, n_{2 k}-2, \ldots, n_{4 k}+1, \ldots, n_{N}\right)\right)
\end{aligned}
$$

where $O_{k}=0$ for $k$ even and $O_{k}=1$ for $k$ odd.

Let us note that by virtue of the substitution

$$
\varphi\left(n_{1}, \ldots, n_{N}\right)=\nu^{E(\sigma)} \tilde{\varphi}\left(n_{1}, \ldots, n_{N}\right)
$$

where $E(\sigma)$ is the number of cycles of even length in the decomposition of $\sigma(15)$, i.e.

$$
E(\sigma)=n_{2}+n_{4}+\ldots
$$

one can get rid of the explicit dependence of $\nu$ from $G L C$ (39). As a result, there are two distinguishing cases, $\nu=0$ and $\nu \neq 0$.

For lower $N$ the conditions $(39)$ take the form

$$
\varphi(0,1)+2 \nu \varphi(2,0)=0
$$

for $N=2(c f .(9))$,

$$
\varphi(1,1,0)+2 \nu \varphi(3,0,0)+\nu \varphi(0,0,1)=0
$$

for $N=3$ and

$$
\begin{aligned}
\varphi(2,1,0,0)+2 \nu \varphi(4,0,0,0)+2 \nu \varphi(1,0,1,0) & =0 \\
\varphi(0,2,0,0)+2 \nu \varphi(2,1,0,0)+2 \nu \varphi(0,0,0,1) & =0 \\
\varphi(0,0,0,1)+4 \nu \varphi(1,0,1,0) & =0
\end{aligned}
$$

for $N=4$. As a result one finds 1-parametric families of solutions for $N=1$ and $N=2$ and 2-parametric families of solutions for $N=3$ and $N=4$.

Let $G_{N}$ be the number of independent solutions of (39). As we show in the next section $G_{N}=\operatorname{dim} T_{S H_{N}(\nu)}$ for all $\nu$. In other words all other conditions on the supertrace do not 
impose any restrictions on the functions $\varphi\left(n_{1}, \ldots, n_{N}\right)$ but merely express supertraces of higher order polynomials of $a_{i}^{\alpha}$ in terms of $\varphi\left(n_{1}, \ldots, n_{N}\right)$.

In the Appendix A we prove the following

Theorem 1: $G_{N}=q(N)$ where $q(N)$ is a number of partitions of $N$ into a sum of odd positive integers, i.e. the number of the solutions of the equation $\sum_{k=0}^{\infty}(2 k+1) n_{k}=N$ for non-negative integers $n_{i}$.

One can guess this result from the particular case of $\nu=0$ where $G L C$ tell us that $\varphi\left(n_{1}, \ldots, n_{N}\right)$ can be nonvanishing (and arbitrary) only when all $n_{2 k}=0$. Interestingly enough, $G_{N}$ remains the same for $\nu \neq 0$.

\section{Supertrace for General Elements}

In this section we prove

Theorem 2: $\operatorname{dim} T_{S H_{N}(\nu)}=G_{N}$ where $G_{N}$ is the number of independent solutions of the ground level conditions (39).

The proof of the Theorem 2 will be given in a constructive way by virtue of the following double induction procedure:

(i). Assuming that $G L C$ are true and $\operatorname{str}\left\{b^{I}, P_{p}(a) \sigma\right\}=0 \forall P_{p}(a), \sigma$ and $I$ provided that $b^{I} \in \mathfrak{B}_{\sigma}$ and

$$
\begin{aligned}
& \lambda(I) \neq-1 ; p \leq k \text { or } \\
& \lambda(I)=-1, E(\sigma) \leq l, p \leq k \text { or } \\
& \lambda(I)=-1 ; p \leq k-2,
\end{aligned}
$$

where $P_{p}(a)$ is an arbitrary degree $p$ polynomial of $a_{i}^{\alpha}(p$ is odd) and $E(\sigma)$ is the number of cycles of even length in the decomposition (15) of $\sigma$, one proves that there exists such a unique extension of the supertrace that the same is true for $l \rightarrow l+1$.

(ii). Assuming that $\operatorname{str}\left\{b^{I}, P_{p}(a) \sigma\right\}=0 \forall P_{p}(a), \sigma$ and $b^{I}$ such that $\sigma(I)=\sigma, p \leq k$ one proves that there exists such a unique extension of the supertrace that the assumption (i) is true for $k \rightarrow k+2$ and $l=0$.

As a result this inductive procedure extends uniquely any solution of $G L C$ to some supertrace on the whole $S H_{N}(\nu)$. (Let us remind ourselves that the supertrace of any odd element of $S H_{N}(\nu)$ is trivially zero by $s l_{2}$ invariance).

The inductive proof of the Theorem 2 is based on the $S_{N}$ covariance of the whole setting and the following important

Lemma 5: Given permutation $\sigma$ which has $E(\sigma)$ cycles of even length in the decomposition (15), the quantity $f^{I J} \sigma$ for $\sigma(I)=\sigma(J)=\sigma$ and $\lambda_{I}=\lambda_{J}=-1$ can be uniquely expanded as $f^{I J} \sigma=\sum_{q} \alpha_{q} \sigma_{q}$ where $\alpha_{q}$ are some coefficients and $E\left(\sigma_{q}\right)=E(\sigma)-1 \forall q$.

Lemma 5 is a simple consequence of the particular form of the structure coefficients $f^{I J}$ (27) and Lemmas 3 and 4. The proof is straightforward. Let us stress that it is Lemma 5 which accounts for the specific properties of the algebra $S H_{N}(\nu)$ in the analysis of this section.

In practice it is convenient to work with the exponential generating functions

$$
\Psi_{\sigma}(\mu)=\operatorname{str}\left(e^{S} \sigma\right), \quad S=\sum_{L=1}^{2 N}\left(\mu_{L} b^{L}\right)
$$


where $\sigma$ is some fixed element of $S_{N}, b^{L} \in \mathfrak{B}_{\sigma}$ and $\mu_{L} \in \mathbb{C}$ are independent parameters. By differentiating over $\mu_{L}$ one can obtain an arbitrary polynomial of $b^{L}$ in front of $\sigma$. The exponential form of the generating functions implies that these polynomials are Weyl ordered. In these terms the induction on a degree of polynomials is equivalent to the induction on a degree of homogeneity in $\mu$ of the power series expansions of $\Psi_{\sigma}(\mu)$.

As a consequence of the general properties discussed in the preceding sections the generating function $\Psi_{\sigma}(\mu)$ must be invariant under the $S_{N}$ similarity transformations

$$
\Psi_{\tau \sigma \tau^{-1}}(\mu)=\Psi_{\sigma}(\tilde{\mu})
$$

where the $S_{N}$ transformed parameters are of the form

$$
\tilde{\mu}_{I}=\sum_{J}\left(\mathfrak{M}\left(\tau \sigma \tau^{-1}\right) \mathfrak{M}^{-1}(\tau) \Lambda^{-1}(\tau) \mathfrak{M}(\tau) \mathfrak{M}^{-1}(\sigma)\right)_{I}^{J} \mu_{J}
$$

and matrices $\mathfrak{M}(\sigma)$ and $\Lambda(\sigma)$ are defined in (22) and (23).

In accordance with the general argument of Section [1] the necessary and sufficient conditions for the existence of even supertrace are the $S_{N^{-}}$-ovariance conditions (45) and the condition that

$$
\operatorname{str}\left\{b^{L},(\exp S) \sigma\right\}=0 \quad \text { for any } \sigma \text { and } L \text {. }
$$

To transform (47) to an appropriate form, let us use the following two general relations which are true for arbitrary operators $X$ and $Y$ and the parameter $\mu \in \mathbb{C}$ :

$$
\begin{aligned}
& X \exp (Y+\mu X)=\frac{\partial}{\partial \mu} \exp (Y+\mu X)+\int t_{2} \exp \left(t_{1}(Y+\mu X)\right)[X, Y] \exp \left(t_{2}(Y+\mu X)\right) D^{1} t \\
& \exp (Y+\mu X) X=\frac{\partial}{\partial \mu} \exp (Y+\mu X)-\int t_{1} \exp \left(t_{1}(Y+\mu X)\right)[X, Y] \exp \left(t_{2}(Y+\mu X)\right) D^{1} t
\end{aligned}
$$

with the convention that

$$
D^{n-1} t=\delta\left(t_{1}+\ldots+t_{n}-1\right) \theta\left(t_{1}\right) \ldots \theta\left(t_{n}\right) d t_{1} \ldots d t_{n} .
$$

The relations (48) and (49) can be derived with the aid of the partial integration (e.g. over $t_{1}$ ) and the following formula

$$
\frac{\partial}{\partial \mu} \exp (Y+\mu X)=\int \exp \left(t_{1}(Y+\mu X)\right) X \exp \left(t_{2}(Y+\mu X)\right) D^{1} t,
$$

which can be proven by expanding in power series. The well-known formula

$$
[X, \exp (Y)]=\int \exp \left(t_{1} Y\right)[X, Y] \exp \left(t_{2} Y\right) D^{1} t
$$

is a consequence of (48) and (49).

With the aid of (48), (49) and (21) one rewrites (47) as

$$
\left(1+\lambda_{L}\right) \frac{\partial}{\partial \mu_{L}} \Psi_{\sigma}(\mu)=\int\left(\lambda_{L} t_{1}-t_{2}\right) \operatorname{str}\left(\exp \left(t_{1} S\right)\left[b^{L}, S\right] \exp \left(t_{2} S\right) \sigma\right) D^{1} t .
$$


This condition should be true for any $\sigma$ and $L$ and plays the central role in the analysis of this section.

There are two essentially distinguishing cases, $\lambda_{L} \neq-1$ and $\lambda_{L}=-1$. In the latter case, the equation (53) takes the form

$$
0=\int \operatorname{str}\left(\exp \left(t_{1} S\right)\left[b^{L}, S\right] \exp \left(t_{2} S\right) \sigma\right) D^{1} t, \quad \lambda_{L}=-1 .
$$

In Appendix B we show by induction that the equations (53) and (54) are consistent in the following sense

$$
\begin{array}{r}
\left(1+\lambda_{K}\right) \frac{\partial}{\partial \mu_{K}} \int\left(\lambda_{L} t_{1}-t_{2}\right) \operatorname{str}\left(\exp \left(t_{1} S\right)\left[b^{L}, S\right] \exp \left(t_{2} S\right) \sigma\right) D^{1} t-(L \leftrightarrow K)=0 \\
\lambda_{L} \neq-1, \lambda_{K} \neq-1
\end{array}
$$

and

$$
\left(1+\lambda_{K}\right) \frac{\partial}{\partial \mu_{K}} \int \operatorname{str}\left(\exp \left(t_{1} S\right)\left[b^{L}, S\right] \exp \left(t_{2} S\right) \sigma\right) D^{1} t=0, \quad \lambda_{L}=-1 .
$$

Note that this part of the proof is quite general and does not depend on a concrete form of the commutation relations of $a_{i}^{\alpha}$ in (2).

By expanding the exponential $e^{S}$ in (44) into power series in $\mu_{K}$ (equivalently $b^{K}$ ) one concludes that the equation (53) uniquely reconstructs the supertrace of monomials containing $b^{K}$ with $\lambda_{K} \neq-1$ (from now on called regular polynomials) via supertraces of some lower order polynomials. The consistency conditions (55) and (56) then guarantee that (53) does not impose any additional conditions on the supertraces of lower degree polynomials and allow one to represent the generating function in the form

$$
\begin{aligned}
\Psi_{\sigma} & =\Phi_{\sigma}(\mu) \\
& +\sum_{L: \lambda_{L} \neq-1} \int_{0}^{1} \frac{\mu_{L} d \tau}{1+\lambda_{L}} \int D^{1} t\left(\lambda_{L} t_{1}-t_{2}\right) \operatorname{str}\left(e^{t_{1}\left(\tau S^{\prime \prime}+S^{\prime}\right)}\left[b^{L},\left(\tau S^{\prime \prime}+S^{\prime}\right)\right] e^{t_{2}\left(\tau S^{\prime \prime}+S^{\prime}\right)} \sigma\right)
\end{aligned}
$$

where we have introduced the generating functions $\Phi_{\sigma}$ for the supertrace of special polynomials, i.e. the polynomials depending only on $b^{L}$ with $\lambda_{L}=-1$,

$$
\Phi_{\sigma}(\mu) \stackrel{\text { def }}{=} \operatorname{str}\left(e^{S^{\prime}} \sigma\right)=\left.\Psi_{\sigma}(\mu)\right|_{\left(\mu_{I}=0 \forall I: \lambda_{I} \neq-1\right)}
$$

and

$$
S^{\prime}=\sum_{L: b^{L} \in \mathfrak{B}_{\sigma}, \lambda_{L}=-1}\left(\mu_{L} b^{L}\right) ; \quad S^{\prime \prime}=S-S^{\prime} .
$$

The relation (57) successively expresses the supertrace of higher order regular polynomials via the supertraces of lower order polynomials.

One can see that the arguments above prove effectively the inductive hypotheses (i) and (ii) for the particular case where either the polynomials $P_{p}(a)$ are regular and/or $\lambda_{I} \neq-1$. Note that for this case the induction on the number of cycles of even length (i) is trivial: one simply proves that a power of polynomial can be increased by two.

Let us now turn to the less trivial case of the special polynomials:

$$
\operatorname{str}\left\{b^{I},\left(\exp S^{\prime}\right) \sigma\right\}=0, \quad \lambda_{I}=-1 .
$$


Consider the part of $\operatorname{str}\left\{b^{I},\left(\exp S^{\prime}\right) \sigma\right\}$ which is of order $k$ in $\mu$ and suppose that $E(\sigma)=$ $l+1$. According to (54) the conditions (60) give

$$
0=\int \operatorname{str}\left(\exp \left(t_{1} S^{\prime}\right)\left[b^{I}, S^{\prime}\right] \exp \left(t_{2} S^{\prime}\right) \sigma\right) D^{1} t .
$$

Substituting $\left[b^{I}, S^{\prime}\right]=\mu^{I}+\nu \sum_{M} f^{I M} \mu_{M}$, where the quantities $f^{I J}$ and $\mu^{I}$ are defined in (25)-(28), one can rewrite the equation (61) in the form

$$
\mu^{I} \Phi_{\sigma}(\mu)=-\nu \int \operatorname{str}\left(\exp \left(t_{1} S^{\prime}\right) \sum_{M} f^{I M} \mu_{M} \exp \left(t_{2} S^{\prime}\right) \sigma\right) D^{1} t
$$

Now we use the inductive hypothesis (i). The right hand side of (62) is a supertrace of at most a degree $k-1$ polynomial of $a_{i}^{\alpha}$ in the sector of degree $k$ polynomials in $\mu$. Therefore one can use the inductive hypothesis (i) to obtain

$$
\int \operatorname{str}\left(\exp \left(t_{1} S^{\prime}\right) \sum_{M} f^{I M} \mu_{M} \exp \left(t_{2} S^{\prime}\right) \sigma\right) D^{1} t=\int \operatorname{str}\left(\exp \left(t_{2} S^{\prime}\right) \exp \left(t_{1} S^{\prime}\right) \sum_{M} f^{I M} \mu_{M} \sigma\right) D^{1} t
$$

where we made use of the simple fact that $\operatorname{str}\left(S^{\prime} F \sigma\right)=-\operatorname{str}\left(F \sigma S^{\prime}\right)=\operatorname{str}\left(F S^{\prime} \sigma\right)$ due to the definition of $S^{\prime}$.

As a result, the inductive hypothesis allows one to transform (60) to the following form

$$
X^{I} \equiv \mu^{I} \Phi_{\sigma}(\mu)+\nu \operatorname{str}\left(\exp \left(S^{\prime}\right) \sum_{M} f^{I M} \mu_{M} \sigma\right)=0 .
$$

By differentiating this equation with respect to $\mu_{J}$ one obtains after symmetrization

$$
\frac{\partial}{\partial \mu_{J}}\left(\mu^{I} \Phi_{\sigma}(\mu)\right)+(I \leftrightarrow J)=-\nu \int \operatorname{str}\left(e^{t_{1} S^{\prime}} b^{J} e^{t_{2} S^{\prime}} \sum_{M} f^{I M} \mu_{M} \sigma\right) D^{1} t+(I \leftrightarrow J) .
$$

An important point is that the system of equations (64) is equivalent to the original equations (63) except for the ground level part $\Phi_{\sigma}(0)$. This can be easily seen from the simple fact that the general solution of the system of equations $\frac{\partial}{\partial \mu_{J}} X^{I}(\mu)+\frac{\partial}{\partial \mu_{I}} X^{J}(\mu)=0$ for entire functions $X^{I}(\mu)$ is of the form $X^{I}(\mu)=X^{I}(0)+\sum_{J} c^{I J} \mu_{J}$ where $X^{I}(0)$ and $c^{J I}=-c^{I J}$ are some constants. The part of (63) linear in $\mu$ is however equivalent to the ground level conditions analyzed in the previous section. Thus (64) contains all information additional to (39). For this reason we will from now on analyze the equation (64).

Using again the inductive hypothesis we move $b^{I}$ to the left and to the right with equal weights to get

$$
\begin{aligned}
& \frac{\partial}{\partial \mu_{J}} \mu^{I} \Phi_{\sigma}(\mu)+(I \leftrightarrow J)=-\frac{\nu}{2} \sum_{M} \operatorname{str}\left(\exp \left(S^{\prime}\right)\left\{b^{J}, f^{I M}\right\} \mu_{M} \sigma\right) \\
& -\frac{\nu}{2} \int \sum_{L, M}\left(t_{1}-t_{2}\right) \operatorname{str}\left(\exp \left(t_{1} S^{\prime}\right) F^{J L} \mu_{L} \exp \left(t_{2} S^{\prime}\right) f^{I M} \mu_{M} \sigma\right) D^{1} t+(I \leftrightarrow J) .
\end{aligned}
$$

The last term on the right hand side of this expression can be shown to vanish under the supertrace operation due to the factor of $\left(t_{1}-t_{2}\right)$, so that one is left with the equation

$$
L^{I J} \Phi_{\sigma}(\mu)=-\frac{\nu}{2} R^{I J}(\mu)
$$


where

$$
R^{I J}(\mu)=\sum_{M} \operatorname{str}\left(\exp \left(S^{\prime}\right)\left\{b^{J}, f^{I M}\right\} \mu_{M} \sigma\right)+(I \leftrightarrow J)
$$

and

$$
L^{I J}=\frac{\partial}{\partial \mu_{J}} \mu^{I}+\frac{\partial}{\partial \mu_{I}} \mu^{J}
$$

The differential operators $L^{I J}$ satisfy the standard $s p(2 E(\sigma))$ commutation relations

$$
\left[L^{I J}, L^{K L}\right]=-\left(\mathcal{C}^{I K} L^{J L}+\mathcal{C}^{I L} L^{J K}+\mathcal{C}^{J K} L^{I L}+\mathcal{C}^{J L} L^{I K}\right)
$$

We show by induction in Appendix 9 that this algebra is consistent with the right-hand side of the basic relation (66) i.e. that

$$
\left[L^{I J}, R^{K L}\right]-\left[L^{K L}, R^{I J}\right]=-\left(\mathcal{C}^{I K} R^{J L}+\mathcal{C}^{J L} R^{I K}+\mathcal{C}^{J K} R^{I L}+\mathcal{C}^{I L} R^{J K}\right)
$$

Generally, these consistency conditions guarantee that the equations (66) express $\Phi_{\sigma}(\mu)$ in terms of $R^{I J}$ in the following way

$$
\Phi_{\sigma}(\mu)=\Phi_{\sigma}(0)+\frac{\nu}{8 E(\sigma)} \sum_{I, J=1}^{2 E(\sigma)} \int_{0}^{1} \frac{d t}{t}\left(1-t^{2 E(\sigma)}\right)\left(L_{I J} R^{I J}\right)(t \mu)
$$

provided that

$$
R^{I J}(0)=0
$$

The latter condition must hold for the consistency of (66) since its left hand side vanishes at $\mu_{I}=0$. In the formula (71) it guarantees that the integral on $t$ converges. In the case under consideration the property $(\sqrt{72})$ is indeed true as a consequence of the definition (67).

Taking into account Lemma 5 and the explicit form of $R^{I J}$ (67) one concludes that the equation (71) expresses uniquely the supertrace of special polynomials via the supertraces of polynomials of lower degrees or via the supertraces of special polynomials of the same degree with a lower number of cycles of even length provided that the $\mu$ independent term $\Phi_{\sigma}(0)$ is an arbitrary solution of $G L C$. This completes the proof of Theorem 2.

Comment 1: The formulae (57) and (71) can be effectively used in practical calculations of supertraces of particular elements of $\mathrm{SH}_{N}(\nu)$.

Comment 2: Any supertrace on $S_{N}(\nu)$ is determined unambiguously in terms of its values on the group algebra of $S_{N}$.

Corollary: Any supertrace on $S H_{N}(\nu)$ is $\rho$-invariant, $\operatorname{str}(\rho(x))=\operatorname{str}(x) \forall x \in S H_{N}(\nu)$, for the antiautomorphism $\rho$ (30).

This is true due to the Comment 2 because $\sigma$ and $\sigma^{-1}=\rho(\sigma)$ belong to the same conjugacy class of $S_{N}$ so that $\operatorname{str}(\rho(\sigma))=\operatorname{str}(\sigma)$. 


\section{Conclusions.}

In this paper we have shown that the algebras $S H_{N}(\nu)$ can be endowed with $q(N)$ independent supertrace operations where $q(N)$ is the number of partitions of $N$ into a sum of odd positive integers. We hope to apply the supertraces constructed in this paper to the analysis of the invariant forms of $S H_{N}(\nu)$. Although a definition of the supertraces on $S H_{N}(\nu)$ behaves regularly with the parameter $\nu$ (in particular, the number of supertraces $q(N)$ is $\nu$ independent) one can expect that this is not the case for the related bilinear forms which can degenerate for some special values of $\nu$ thus giving rise to ideals of $S H_{N}(\nu)$ as it happens [14] for the simplest case of $N=2$. The analysis of the structure of these ideals is a challenging problem important for various application of $S H_{N}(\nu)$, including analysis of its representations. We are going to study this problem for some lower values of $N>2$ in the future publication.

In conclusion let us note that the method of the analysis of supertraces presented in this paper is rather general. Practically, the only information of the specific structure of $S H_{N}(\nu)$ is that Lemma 5 is true. Hopefully one can use the analogous methods for the analysis of supertraces of other associative algebras.

\section{Acknowledgements}

Authors are very grateful to M. Soloviev for useful discussions. The research described in this publication was made possible in part by Grant No MQM300 from the International Science Foundation and Government of Russian Federation. This work was supported in part by the Russian Basic Research Foundation, grant 93-02-15541, and INTAS grant 93-0633.

\section{APPENDICES}

\section{A Independence $G_{N}$ of $\nu$.}

For the case $\nu=0$ it was argued in section IV that (39) possesses $q(N)$ independent solutions. Let us now consider the case $\nu \neq 0$. By induction on a number of cycles of even length $e=E(\sigma)$ we show that given $\sigma$ with $E(\sigma)=e \geq 1$ there is only one independent equation on $\operatorname{str}(\sigma)$ provided that all equations (39) with $E(\sigma)=e^{\prime}<e$ are resolved. In this proof we set $\nu=1$ that does not lead to the loss of generality due to the scaling property (40). The first step of the induction consists of the observation that there are no equations for the case $E(\sigma)=0$.

Let us consider the case where there are two equations (38) on $\operatorname{str}(\sigma)$ for some $\sigma$. This is only possible if $\sigma=c_{1} c_{2} \sigma^{\prime}$ where $c_{1}$ and $c_{2}$ are some cycles in the decomposition of $\sigma$ such that $\left|c_{1}\right|=2 k,\left|c_{2}\right|=2 l$ and $k \neq l$. Note that $E\left(\sigma^{\prime}\right)=E(\sigma)-2=e-2$.

Without loss of generality let us set

$$
\begin{gathered}
c_{1}=K_{12} K_{23} \ldots K_{(2 k-1) 2 k}, \quad c_{2}=K_{(2 k+1)(2 k+2) \ldots K_{(2 k+2 l-1)(2 k+2 l)},} \\
b_{1}^{\alpha}=\frac{1}{\sqrt{2 k}}\left(a_{1}^{\alpha}-a_{2}^{\alpha}+\ldots-a_{2 k}^{\alpha}\right), \quad b_{2}^{\alpha}=\frac{1}{\sqrt{2 l}}\left(a_{2 k+1}^{\alpha}-a_{2 k+1}^{\alpha}+\ldots-a_{2 k+2 l}^{\alpha}\right) .
\end{gathered}
$$


Also we introduce

$$
c=K_{1(2 k+1)} c_{1} c_{2}=K_{12} K_{23} \ldots K_{(2 k+2 l-1)(2 k+2 l)}
$$

and

$$
b^{\alpha}=\frac{1}{\sqrt{2 k+2 l}}\left(\sqrt{2 k} b_{1}^{\alpha}+\sqrt{2 l} b_{2}^{\alpha}\right)=\frac{1}{\sqrt{2 k+2 l}}\left(a_{1}^{\alpha}-a_{2}^{\alpha}+\ldots-a_{2 k+2 l}^{\alpha}\right) .
$$

The corresponding equations (38) take the form

$$
\operatorname{str}(\sigma)=-\operatorname{str}\left(\left(\left[b_{1}^{0}, b_{1}^{1}\right]-1\right) \sigma\right)
$$

and

$$
\operatorname{str}(\sigma)=-\operatorname{str}\left(\left(\left[b_{2}^{0}, b_{2}^{1}\right]-1\right) \sigma\right) .
$$

Using the following simple identity which holds for any trace on $S_{N}$,

$$
\operatorname{str}\left(\frac{1}{2 k} \sum_{p=1}^{2 k} \sum_{q=2 k+1}^{2 k+2 l} K_{p q} \sigma\right)=2 l \operatorname{str}\left(K_{1(2 k+1)} \sigma\right)
$$

one can rewrite the right hand side of (A5) as

$$
\operatorname{str}\left(\left(\left[b_{1}^{0}, b_{1}^{1}\right]-1\right) \sigma\right)=\operatorname{str}\left(\left(\left[b_{1}^{0}, b_{1}^{1}\right]-1-\frac{1}{2 k} \sum_{p=1}^{2 k} \sum_{q=2 k+1}^{2 k+2 l} K_{p q}\right) \sigma\right)+2 l \operatorname{str}\left(K_{1(2 k+1)} \sigma\right) .
$$

The direct analysis based on the commutation relations (2) and (3) then shows that the first term on the right hand side of (A7) is the supertrace of a linear combination of permutations which all contain the cycle $c_{2}$ in their decompositions. The second term is the supertrace of the permutation which contains the cycle $c$ ( $\mathrm{A3}$ ) in its decomposition. It is easy to see that for each of these terms the number of cycles of even length is $E(\sigma)-1$. This allows us to apply the equation (38) to each of this terms due to the inductive hypothesis. We identify $c_{0}$ with $c_{2}$ and $c$, respectively, for the first and second terms on the right hand side of (A7). As a result the equation (A5) turns out to be transformed to the form

$$
\begin{aligned}
\operatorname{str}(\sigma) & =\operatorname{str}\left(\left(\left[b_{2}^{0}, b_{2}^{1}\right]-1\right)\left(\left[b_{1}^{0}, b_{1}^{1}\right]-1-\frac{1}{2 k} \sum_{p=1}^{2 k} \sum_{q=2 k+1}^{2 k+2 l} K_{p q}\right) \sigma\right) \\
& +\operatorname{str}\left(\left(\left[b^{0}, b^{1}\right]-1\right) 2 l K_{1(2 k+1)} \sigma\right)
\end{aligned}
$$

Analogously one obtains for (A6)

$$
\begin{aligned}
\operatorname{str}(\sigma) & =\operatorname{str}\left(\left(\left[b_{1}^{0}, b_{1}^{1}\right]-1\right)\left(\left[b_{2}^{0}, b_{2}^{1}\right]-1-\frac{1}{2 l} \sum_{p=1}^{2 k} \sum_{q=2 k+1}^{2 k+2 l} K_{p q}\right) \sigma\right) \\
& +\operatorname{str}\left(\left(\left[b^{0}, b^{1}\right]-1\right) 2 k K_{1(2 k+1)} \sigma\right) .
\end{aligned}
$$


Let us prove that the difference of the right hand sides of (A8) and (A9) vanishes. With the aid of the simple consequence of the $S_{N}$ invariance

$$
\frac{1}{4 k l} \operatorname{str}\left(\left[b_{i}^{0}, b_{i}^{1}\right] \sum_{p=1}^{2 k} \sum_{q=2 k+1}^{2 k+2 l} K_{p q} \sigma\right)=\operatorname{str}\left(\left[b_{i}^{0}, b_{i}^{1}\right] K_{1(2 k+1)} \sigma\right), \quad i=1,2
$$

this difference can be transformed to the form

$$
X=\operatorname{str}\left(\left(2 k\left[b_{1}^{0}, b_{1}^{1}\right]-2 l\left[b_{2}^{0}, b_{2}^{1}\right]\right) K_{1(2 k+1)} \sigma\right),
$$

where we have taken into account that

$$
\operatorname{str}\left(\left[b^{0}, b^{1}\right] K_{1(2 k+1)} \sigma\right)=0
$$

as a consequence of the inductive hypothesis and $G L C$ (37) and that

$$
\operatorname{str}\left(\left[\left(\left[b_{2}^{0}, b_{2}^{1}\right]-1\right),\left(\left[b_{1}^{0}, b_{1}^{1}\right]-1\right)\right] \sigma\right)=0
$$

since each term in the commutator belongs to the group algebra of $S_{N}$ and commutes with $\sigma$ so that (A12) vanishes for any supertrace on the group algebra of $S_{N}$.

Using the relation $b_{1}^{\alpha}=1 / \sqrt{2 k}\left(\sqrt{2 k+2 l} b^{\alpha}-\sqrt{2 l} b_{2}^{\alpha}\right)$ one transforms $X$ to the form

$$
X=2 \operatorname{str}\left(\left((k+l)\left[b^{0}, b^{1}\right]-\sqrt{l(k+l)}\left[b^{0}, b_{2}^{1}\right]-\sqrt{l(k+l)}\left[b_{2}^{0}, b^{1}\right]\right) K_{1(2 k+1)} \sigma\right) .
$$

Due to the $S_{N}$ invariance the second term on the right hand side of (A13) can be rewritten as

$$
\begin{aligned}
& -2 \sqrt{l(k+l)} \operatorname{str}\left(\left[b^{0}, b_{2}^{1}\right] K_{1(2 k+1)} \sigma\right)=-\frac{\sqrt{l(k+l)}}{k+l} \sum_{p=1}^{2 k+2 l} \operatorname{str}\left(c^{p}\left[b^{0}, b_{2}^{1}\right] c^{-p} K_{1(2 k+1)} \sigma\right) \\
= & -\frac{\sqrt{l}}{\sqrt{k+l}} \operatorname{str}\left(\left[b^{0}, \sum_{p=1}^{2 k+2 l}(-1)^{p} c^{p} b_{2}^{1} c^{-p}\right] K_{1(2 k+1)} \sigma\right)=-2 l \operatorname{str}\left(\left[b^{0}, b^{1}\right] K_{1(2 k+1)} \sigma\right) .(\mathrm{A} 14)
\end{aligned}
$$

Analogously one can transform the third term on the right hand side of (A13). Using again (A11) one concludes that $X=0$.

Thus it is shown that the number of solutions of (39) is equal to the number of the conjugacy classes in $S_{N}$ with $E(\sigma)=0$. This completes the proof of Theorem 1.

\section{B Consistency for $\lambda \neq-1$}

Let us prove by induction that the equations (55) are true for any two $\mu_{1} \equiv \mu_{K_{1}}$ and $\mu_{2} \equiv \mu_{K_{2}}$ such that both $\lambda_{1} \equiv \lambda_{K_{1}} \neq-1$ and $\lambda_{2} \equiv \lambda_{K_{2}} \neq-1$. To implement induction one selects from (47) a part of order $k$ in $\mu$ and observes that it contains the anticommutator of $b^{L}$ with a degree $k$ polynomial in $b^{M}$ while the part on the right hand side of the differential version (53) of (47) which is of the same order in $\mu$ has the order $k-1$ as the polynomial of $b^{M}$. This happens because of the presence of the commutator $\left[b^{L}, S\right]$ 
which is a degree zero polynomial due to the basic relations (2), (3). As a result, the inductive hypothesis allows one to use the properties of the supertrace provided that the above commutator is always handled as the right hand side of (2) (i.e. it is not allowed to represent it again as a difference of the second-order polynomials).

Direct differentiation with the aid of (51) gives

$$
\begin{array}{r}
\left(1+\lambda_{2}\right) \frac{\partial}{\partial \mu_{2}} \int\left(\lambda_{1} t_{1}-t_{2}\right) \operatorname{str}\left(e^{t_{1} S}\left[b^{1}, S\right] e^{t_{2} S} \sigma\right) D^{1} t-(1 \leftrightarrow 2)= \\
\left(\int\left(1+\lambda_{2}\right)\left(\lambda_{1} t_{1}-t_{2}\right) \operatorname{str}\left(e^{t_{1} S}\left[b^{1}, b^{2}\right] e^{t_{2} S} \sigma\right) D^{1} t-(1 \leftrightarrow 2)\right)+ \\
\left(\int\left(1+\lambda_{2}\right)\left(\lambda_{1}\left(t_{1}+t_{2}\right)-t_{3}\right) \operatorname{str}\left(e^{t_{1} S} b^{2} e^{t_{2} S}\left[b^{1}, S\right] e^{t_{3} S}\right) D^{2} t-(1 \leftrightarrow 2)\right)+ \\
\left(\int\left(1+\lambda_{2}\right)\left(\lambda_{1} t_{1}-t_{2}-t_{3}\right) \operatorname{str}\left(e^{t_{1} S}\left[b^{1}, S\right] e^{t_{2} S} b^{2} e^{t_{3} S} \sigma\right) D^{2} t-(1 \leftrightarrow 2)\right) .
\end{array}
$$

We have to show that the right hand side of $(\mathbb{B 1})$ vanishes. Let us first transform the second and the third terms on the right-hand side of (B1). The idea is to move the operators $b^{2}$ through the exponentials towards the commutator $\left[b^{1}, S\right]$ so that to use then Jacobi identities for the double commutators. This can be done in two different ways inside the supertrace so that one has to fix appropriate weight factors for each of these processes. The correct weights turn out to be

$$
\begin{array}{r}
D^{2} t\left(\lambda_{1}\left(t_{1}+t_{2}\right)-t_{3}\right) b^{2} \equiv D^{2} t\left(\lambda_{1}-t_{3}\left(1+\lambda_{1}\right)\right) b^{2}= \\
D^{2} t\left(\left(\frac{\lambda_{1} \lambda_{2}}{1+\lambda_{2}}-t_{3}\left(1+\lambda_{1}\right)\right) \overrightarrow{b^{2}}+\frac{\lambda_{1}}{1+\lambda_{2}} \overleftarrow{b^{2}}\right)
\end{array}
$$

and

$$
\begin{array}{r}
D^{2} t\left(\lambda_{1} t_{1}-t_{2}-t_{3}\right) b^{2} \equiv D^{2} t\left(\left(\lambda_{1}+1\right) t_{1}-1\right) b^{2}= \\
D^{2} t\left(\left(t_{1}\left(1+\lambda_{1}\right)-\frac{1}{1+\lambda_{2}}\right) \overleftarrow{b^{2}}-\frac{\lambda_{2}}{1+\lambda_{2}} \overrightarrow{b^{2}}\right)
\end{array}
$$

in the second and third terms on the right hand side of (B1), respectively. Here the notations $\vec{A}$ and $\overleftarrow{A}$ imply that the operator $A$ has to be moved from its position to the right and to the left, respectively. Using (52) along with the simple formula

$$
\int \phi\left(t_{3}, \ldots t_{n+1}\right) D^{n} t=\int t_{1} \phi\left(t_{2}, \ldots t_{n}\right) D^{n-1} t
$$

one finds that all terms which involve both $\left[b^{1}, S\right]$ and $\left[b^{2}, S\right]$ cancel pairwise after antisymmetrization $1 \leftrightarrow 2$.

As a result, one is left with some terms involving double commutators which by virtue of Jacobi identities and antisymmetrization all reduce to

$$
\int\left(\lambda_{1} \lambda_{2} t_{1}+t_{2}-t_{1} t_{2}\left(1+\lambda_{1}\right)\left(1+\lambda_{2}\right)\right) \operatorname{str}\left(\exp \left(t_{1} S\right)\left[S,\left[b^{1}, b^{2}\right]\right] \exp \left(t_{2} S\right) \sigma\right) D^{1} t .
$$


Finally one observes that this expression can be equivalently rewritten in the form

$$
\int\left(\lambda_{1} \lambda_{2} t_{1}+t_{2}-t_{1} t_{2}\left(1+\lambda_{1}\right)\left(1+\lambda_{2}\right)\right)\left(\frac{\partial}{\partial t_{1}}-\frac{\partial}{\partial t_{2}}\right) \operatorname{str}\left(\exp \left(t_{1} S\right)\left[b^{1}, b^{2}\right] \exp \left(t_{2} S\right) \sigma\right) D^{1} t
$$

and after integration by parts cancel the first term on the right-hand side of (B1). Thus it is shown that the equations (53) are mutually compatible for the case $\lambda_{1,2} \neq-1$.

Analogously one can show that the equations (53) are consistent with (54). Actually, let $\lambda_{1}=-1, \lambda_{2} \neq-1$. Let us prove that

$$
\frac{\partial}{\partial \mu_{2}} \operatorname{str}\left(\left[b^{1}, \exp (S)\right] \sigma\right)=0
$$

provided that the supertrace is well defined for the lower order polynomials. The explicit differentiation gives

$$
\begin{aligned}
\frac{\partial}{\partial \mu_{2}} \operatorname{str}\left(\left[b^{1}, \exp (S)\right] \sigma\right) & =\int \operatorname{str}\left(\left[b^{1}, \exp \left(t_{1} S\right) b^{2} \exp \left(t_{2} S\right)\right] \sigma\right) D^{1} t \\
& =\left(1+\lambda_{2}\right)^{-1} \operatorname{str}\left(\left[b^{1},\left(b^{2} \exp (S)+\lambda_{2} \exp (S) b^{2}\right)\right] \sigma\right)+\ldots
\end{aligned}
$$

where dots denote some terms of the form $\operatorname{str}\left(\left[b^{1}, B\right] \sigma\right)$ involving further commutators inside $B$, which therefore amount to some lower order polynomials and vanish by the inductive hypothesis. As a result, one finds

$$
\begin{aligned}
\frac{\partial}{\partial \mu_{2}} \operatorname{str}\left(\left[b^{1}, \exp (S)\right] \sigma\right) \quad & =\left(1+\lambda_{2}\right)^{-1} \operatorname{str}\left(\left(b^{2}\left[b^{1}, \exp (S)\right]+\lambda_{2}\left[b^{1}, \exp (S)\right] b^{2}\right) \sigma\right) \\
& +\left(1+\lambda_{2}\right)^{-1} \operatorname{str}\left(\left(\left[b^{1}, b^{2}\right] \exp (S)+\lambda_{2} \exp (S)\left[b^{1}, b^{2}\right]\right) \sigma\right),
\end{aligned}
$$

which expression vanishes by the inductive hypothesis too.

\section{Consistency for $\lambda=-1$}

In order to prove $(70)$ we use the inductive hypothesis (i). In this appendix we use the convention that any upper or lower indices denoted by the same letter are automatically symmetrized, e.g. $F^{I I} \stackrel{\text { def }}{=} \frac{1}{2}\left(F^{I_{1} I_{2}}+F^{I_{2} I_{1}}\right)$. Let us write the identity

$$
0=\sum_{M} \operatorname{str}\left(\left[\exp \left(S^{\prime}\right)\left\{b^{I}, f^{I M}\right\} \mu_{M}, b^{J} b^{J}\right] \sigma\right)-(I \leftrightarrow J)
$$

which holds due to Lemma 5 for all terms of degree $k-1$ in $\mu$ with $E(\sigma) \leq l+1$ and for all lower order polynomials in $\mu$ (one can always move $f^{I J}$ to $\sigma$ combining them into a combination of elements of $S_{N}$ analyzed in Lemma 5).

The straightforward calculation of the commutator on the right-hand-side of (C1) gives $0=X_{1}+X_{2}+X_{3}$, where

$$
\begin{aligned}
& X_{1}=-\sum_{M, L} \int \operatorname{str}\left(\exp \left(t_{1} S^{\prime}\right)\left\{b^{J}, F^{J L}\right\} \mu_{L} \exp \left(t_{2} S^{\prime}\right)\left\{b^{I}, f^{I M}\right\} \mu_{M} \sigma\right) D^{1} t-(I \leftrightarrow J), \\
& X_{2}=\sum_{M} \operatorname{str}\left(\exp \left(S^{\prime}\right)\left\{\left\{b^{J}, F^{I J}\right\}, f^{I M}\right\} \mu_{M} \sigma\right)-(I \leftrightarrow J), \\
& X_{3}=\sum_{M} \operatorname{str}\left(\exp \left(S^{\prime}\right)\left\{b^{I},\left\{b^{J},\left[f^{I M}, b^{J}\right]\right\}\right\} \mu_{M} \sigma\right)-(I \leftrightarrow J) .
\end{aligned}
$$


The terms bilinear in $f$ in $X_{1}$ cancel due to the antisymmetrization $(I \leftrightarrow J)$ and the inductive hypothesis (i). As a result, one can transform $X_{1}$ to the form

$$
X_{1}=\left(-\frac{1}{2}\left[L^{J J}, R^{I I}\right]+2 \operatorname{str}\left(e^{S^{\prime}}\left\{b^{I}, f^{I J}\right\} \mu^{J} \sigma\right)\right)-(I \leftrightarrow J) .
$$

Substituting $F^{I J}=\mathcal{C}^{I J}+\nu f^{I J}$ and $f^{I M}=\nu^{-1}\left(\left[b^{I}, b^{M}\right]-\mathcal{C}^{I M}\right)$ one transforms $X_{2}$ to the form

$$
X_{2}=2 \mathcal{C}^{I J} R^{I J}-2\left(\operatorname{str}\left(e^{S^{\prime}}\left\{b^{J}, f^{I J}\right\} \mu^{I} \sigma\right)-(I \leftrightarrow J)\right)+Y
$$

where

$$
Y=\operatorname{str}\left(e^{S^{\prime}}\left\{\left\{b^{J}, f^{I J}\right\},\left[b^{I}, S^{\prime}\right]\right\} \sigma\right)-(I \leftrightarrow J)
$$

Using that

$$
\operatorname{str}\left(\exp \left(S^{\prime}\right)\left[P f^{I J} Q, S^{\prime}\right] \sigma\right)=0
$$

provided that the inductive hypothesis can be used, one transforms $Y$ to the form

$$
\begin{aligned}
Y=\operatorname{str}\left(e^{S^{\prime}}(\right. & -\left[f^{I J},\left(b^{I} S^{\prime} b^{J}+b^{J} S^{\prime} b^{I}\right)\right]-b^{I}\left[f^{I J}, S^{\prime}\right] b^{J}-b^{J}\left[f^{I J}, S^{\prime}\right] b^{I} \\
& \left.\left.+\left[f^{I J},\left\{b^{I}, b^{J}\right\}\right] S^{\prime}\right) \sigma\right) .
\end{aligned}
$$

Let us rewrite $X_{3}$ in the form $X_{3}=X_{3}^{s}+X_{3}^{a}$ where

$$
\begin{aligned}
& X_{3}^{s}=\frac{1}{2} \sum_{M} \operatorname{str}\left(e^{S^{\prime}}\left(\left\{b^{I},\left\{b^{J},\left[f^{I M}, b^{J}\right]\right\}\right\}+\left\{b^{J},\left\{b^{I},\left[f^{I M}, b^{J}\right]\right\}\right\}\right) \mu_{M} \sigma\right)-(I \leftrightarrow J) \\
& X_{3}^{a}=\frac{1}{2} \sum_{M} \operatorname{str}\left(e^{S^{\prime}}\left(\left\{b^{I},\left\{b^{J},\left[f^{I M}, b^{J}\right]\right\}\right\}-\left\{b^{J},\left\{b^{I},\left[f^{I M}, b^{J}\right]\right\}\right\}\right) \mu_{M} \sigma\right)-(I \leftrightarrow J) .
\end{aligned}
$$

With the aid of the Jacobi identities $\left[f^{I M}, b^{J}\right]-\left[f^{J M}, b^{I}\right]=\left[f^{I J}, b^{M}\right]$ one expresses $X_{3}^{s}$ in the form

$$
X_{3}^{s}=\frac{1}{2} \operatorname{str}\left(e^{S^{\prime}}\left(\left\{b^{I}, b^{J}\right\}\left[f^{I J}, S^{\prime}\right]+\left[f^{I J}, S^{\prime}\right]\left\{b^{I}, b^{J}\right\}+2 b^{I}\left[f^{I J}, S^{\prime}\right] b^{J}+2 b^{J}\left[f^{I J}, S^{\prime}\right] b^{I}\right) \sigma\right) .
$$

$X_{3}^{a}$ can be transformed to the form

$$
X_{3}^{a}=\frac{1}{2} \sum_{M} \operatorname{str}\left(e^{S^{\prime}}\left[F^{I J},\left[f^{I M}, b^{J}\right]\right] \mu_{M} \sigma\right)-(I \leftrightarrow J) .
$$

By virtue of the substitutions $F^{I J}=\mathcal{C}^{I J}+\nu f^{I J}$ and $f^{I M}=\nu^{-1}\left(\left[b^{I}, b^{M}\right]-\mathcal{C}^{I M}\right)$ in (C8) one finds after simple transformations that $Y+X_{3}=0$. From (C3) and (C4) it follows then that the right hand side of (C1) equals to $\frac{1}{2}\left(\left[L^{I I}, R^{J J}\right]-\left[L^{J J}, R^{I I}\right]\right)+2 \mathcal{C}^{I J} R^{I J}$. This completes the proof of the consistency conditions $(70)$. 


\section{References}

[1] A. Polychronakos, Phys. Rev. Lett. 69 (1992) 703.

[2] L. Brink, H. Hansson and M.A. Vasiliev, Phys. Lett. B286 (1992) 109.

[3] L. Brink, H. Hansson, S.E. Konstein and M.A. Vasiliev, Nucl. Phys. B401 (1993) 591.

[4] F. Calogero, J. Math. Phys., 10 (1969) 2191, 2197; ibid 12 (1971) 419.

[5] L. Brink and M.A. Vasiliev, Mod. Phys. Lett. A8 (1993) 3585.

[6] C.F.Dunkl, Trans. Am. Math. Soc. 311 (1989) 167.

[7] C.N. Pope, L.J. Romans and X. Shen, Phys. Lett. B236 (1990) 173; Phys. Lett. B242 (1990) 401.

[8] E. Bergshoeff and M.A. Vasiliev, Int. J. Mod. Phys. A10 (1995) 3477.

[9] A. Capelli, C. Trugenberger and G. Zemba, Nucl. Phys. B396 (1993) 465;

S. Iso, D. Karabali and B. Sakita, Phys. Lett. B296 (1992) 143.

[10] M.A. Vasiliev, Phys. Lett. B285 (1992) 225, Class. Quant. Grav. 11 (1994) 649 (and references therein).

[11] Y. Yamagishi, Phys. Lett. B259 (1991) 436;

F. Yu and Y.-S. Wu, Phys. Lett. B263 (1991) 220.

[12] J. M. Leinaas and J. Myrheim, Phys. Rev. B37 (1988) 9286.

[13] J. Moyal, Proc. Camb. Phil. Soc. 45 (1949) 99;

A. Kirillov, Elements of the Theory of Representations, Nauka, Moscow 1978 (in Russian);

M.A. Vasiliev, Fortschr. Phys. 36 (1988) 33.

[14] M.A. Vasiliev, JETP Letters, 50 (1989) 344-347; Int. J. Mod. Phys. A6 (1991) 1115.

[15] E. Bergshoeff, B. de Wit and M.A. Vasiliev, Nucl. Phys. B366 (1991) 315.

[16] M.A. Vasiliev, Annals of Phys. 190 (1989) 59;

S.E. Konstein and M.A. Vasiliev, Nucl. Phys. B331 (1990) 475.

[17] G.E.Andrews, The Theory of Partitions, Encyclopedia of Math. and its Appl., ed. by G.-C. Rota, vol.2, Addison-Wesley Publ. (1976). 